\title{
What Are the 30-day Readmission Rates Across Orthopaedic Subspecialties?
}

\author{
James T. Bernatz BS, Jonathan L. Tueting MD, Scott Hetzel MS, \\ Paul A. Anderson MD
}

Received: 15 July 2015/Accepted: 13 October 2015/Published online: 26 October 2015

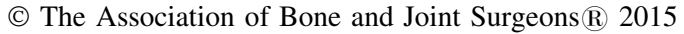

\begin{abstract}
Background The Centers for Medicare \& Medicaid Services (CMS) now include hip and knee replacements in the Hospital Readmission Reduction Program. The 30-day readmission rate is an important quality metric; however, the incidence has not yet been defined across the numerous orthopaedic subspecialties. Elucidating the readmission rate for each subspecialty may indicate that certain services are being disincentivized by the CMS reimbursement program. Furthermore, the "planned" and "unplanned" definitions of readmission have not been well examined to determine their

Each author certifies that he or she, or a member of his or her immediate family, has no funding or commercial associations (eg, consultancies, stock ownership, equity interest, patent/licensing arrangements, etc) that might pose a conflict of interest in connection with the submitted article.

All ICMJE Conflict of Interest Forms for authors and Clinical Orthopaedics and Related Research ${ }^{\mathbb{R}}$ editors and board members are on file with the publication and can be viewed on request.

Each author certifies that his or her institution approved the human protocol for this investigation, that all investigations were conducted in conformity with ethical principles of research, and that informed consent for participation in the study was obtained.

This work was performed at the Department of Orthopedics and Rehabilitative Medicine, University of Wisconsin School of Medicine and Public Health, Madison, WI, USA.
\end{abstract}

J. T. Bernatz, J. L. Tueting, P. A. Anderson

University of Wisconsin School of Medicine and Public Health, Madison, WI, USA

S. Hetzel

Department of Biostatistics and Medical Informatics, University of Wisconsin-Madison, Madison, WI, USA

J. T. Bernatz (ه)

UW Medical Foundation Centennial Building, 1685 Highland

Avenue, 6th Floor, Madison, WI 53705-2281, USA

e-mail: jbernatz@wisc.edu clinical relevance and representation of safe patient care. Therefore, reducing the 30-day readmission rate has become a top priority in orthopaedic quality assurance.

Questions/purposes (1) What are the 30-day readmission rates for the different orthopaedic subspecialties? (2) What are the risk factors associated with readmission within 30 days? (3) What are the causes of 30-day readmissions? (4) What is the interrater agreement among CMS, hospital, and clinician definitions of planned and unplanned readmissions? Methods We retrospectively examined one tertiary care academic hospital's quality improvement database and identified 4792 discharges from the department of orthopaedics during a continuous 24-month period. Discharges were divided and analyzed according to the subspecialty of orthopaedic care. Demographics and comorbidities were extracted from the database and subjected to univariate and multivariate analysis to determine risk factors for 30-day readmission. Further chart review was conducted on all cases of 30-day readmission to identify causes. The authors' determination of planned versus unplanned was compared with two other definitions (hospital and CMS) and analyzed for agreement by using Fleiss' kappa for multiple rater.

Results The all-cause 30-day readmission rate was $4 \%$ (95\% confidence interval [CI], 3.8-4.8). The unplanned readmission rate was $3 \%(95 \% \mathrm{CI}, 2.8-3.8)$. After controlling for relevant confounding variables, we found that length of stay (odds ratio [OR], 1.10 per day; $\mathrm{p}<0.001$ ), American Society of Anesthesiologists score (OR, 1.89 per point; $\mathrm{p}<0.001$ ), and care under trauma (OR, 2.55; $\mathrm{p}<$ $0.001)$ or "other" $(\mathrm{OR}, 1.65 ; \mathrm{p}=0.009)$ as compared with joint subspecialty were associated with increased risk of readmission. Of the 160 unplanned readmissions, 93 (58\%) were surgical and $67(42 \%)$ were medical. The most common surgical cause was surgical site infection $(38 \%$ of surgical readmissions) and the most common medical 
causes were gastrointestinal bleed, pulmonary embolus, and unrelated trauma (each $9 \%$ of medical readmissions). There was poor agreement (Fleiss' kappa $=0.120$ ) among the three definitions of planned readmission.

Conclusions There are important differences in the risk of readmission by subspecialty across orthopaedics and the CMS-driven disincentives may be applied unequally across these subspecialties. This could result in hospitals deemphasizing those service lines and could potentially limit access to care for the patients most in need. Avenues of readmission reduction should be further studied including telephone followup programs and outpatient management of threatened wounds. Clinical, hospital, and CMS definitions of planned readmission have poor agreement, suggesting that hospitals are being unnecessarily penalized. The CMS should develop a more clinically relevant definition of 30-day readmission to more accurately evaluate the rate of readmissions.

Level of Evidence Level III, therapeutic study.

\section{Introduction}

Healthcare reform aims to increase value of care, which is accomplished by decreasing costs and/or improving patient outcomes. The Centers for Medicare \& Medicaid Services (CMS) have identified 30-day readmission as an adverse event that represents both increased cost and poorer outcome [15]. In 2008, 20\% of all hospitalized Medicare patients were readmitted within 30 days, costing USD 17 billion according to the Medicare Payment Advisory Committee [21]. The CMS now collects and reports 30-day readmission rates as an indicator of quality, and the Patient Protection and Affordable Care Act distributes monetary penalties to hospitals with readmission rates that exceed a national benchmark [12]. These penalties were initially enacted for three conditions (myocardial infarction, heart failure, and pneumonia) but they were expanded in 2014 to include hip and knee replacements [5]. Facilities in the worst quartile, after adjusting for patient population, will lose reimbursement dollars. With these changes, the 30-day readmission rate has become an important quality assurance measure in orthopaedics.

Orthopaedic subspecialists treat diverse patient populations with varying intensities of care. These factors could contribute to different 30-day readmission rates based on subspecialty. Previous studies have shown a range of 30day readmission rate from $3 \%$ for joint arthroplasty to $14 \%$ for spinal deformity surgery $[26,28]$. If there are differences across orthopaedic subspecialties in readmission rates, then CMS-driven disincentives may be applied unequally across subspecialties. This could result in hospitals deemphasizing those service lines and, to the degree that some of these services (such as complex spine care or Level I trauma) are available mainly at tertiary care hospitals, those disincentives could result in limiting access to care to the neediest patients. Previous studies have identified risk factors for readmission in single institutions [9, 26, 28]. It has been demonstrated that risk factors for readmission vary according to patient population [2]; therefore, we aim to characterize the readmission risk factors in our large, academic, tertiary care hospital to add to the body of published data. We also want to determine the causes of readmission so that future studies may be directed at quality improvement initiatives to minimize the identified causes. Finally, planned readmissions are not subject to loss of payment by CMS, whereas unplanned readmissions are. There are several definitions for unplanned readmission but, to our knowledge, the degree to which these definitions agree with one another is unknown. This determination is important in an era in which unplanned readmissions may go unreimbursed by payers.

We therefore asked: (1) What are the 30-day readmission rates for the different orthopaedic subspecialties? (2) What are the risk factors associated with readmission within 30 days? (3) What are the causes of 30-day readmission? (4)What is the interrater agreement among CMS, hospital, and clinician definitions of planned and unplanned readmissions?

\section{Patients and Methods}

We reviewed data from all patients who were discharged from the Department of Orthopaedics at the University of Wisconsin Hospitals and Clinics (UWHC) between April 1, 2012, and March 31, 2014, yielding 4792 unique discharges. These encounters were identified using the hospital's quality improvement database, which did not track readmission to outside hospitals or readmissions for scheduled chemotherapy.

Included patients were those discharged from the service of an attending orthopaedic surgeon. The index admission could have been for either surgical or nonsurgical care. Patients who were readmitted to UWHC 30 days inclusive of discharge were included in the readmission cohort. These included same-day readmissions. There were no restrictions on service or provider during the readmission. Both planned and unplanned readmissions were included.

Variables of interest were extracted including age, sex, heritage (self-reported), insurance (Medicare, Medicaid, or private), body mass index, and American Society of Anesthesiologists (ASA) score. Further data included the information from the index admission (date of admission, attending provider, date of discharge, discharge disposition) and 30-day readmission if applicable (date of 
readmission, cause of readmission based on International Classification of Diseases, 9th Revision codes, planned/ unplanned readmission based on hospital and CMS definitions).

Causes of readmission, as determined by the reviewers, were categorized as planned, medical, or surgical, similar to previous studies on this topic [9]. This was taken from the principal admitting diagnosis on readmission. The medical readmissions were subdivided into body systems, and surgical readmissions were categorized as medication/ equipment-related, surgical site-related, and procedure-related. We categorized each surgeon in the department according to their subspecialty, thus allocating each discharge to a certain subspecialty. Discharge disposition was documented as to home, home with home health care, skilled nursing facility (SNF), or other (hospice, long-term acute care hospital, or jail).

Authors reviewed each readmitted patient's medical record to determine the cause of the readmission and whether the readmission was planned. Three definitions were applied to determine planned versus unplanned readmission: CMS, hospital, and clinical. The CMS defines planned readmission based on type of care and acuity of illness: (1) a few specific, limited types of care are always considered planned (obstetric delivery, transplant surgery, maintenance chemotherapy/radiotherapy/immunotherapy, rehabilitation); (2) otherwise, a planned readmission is defined as a nonacute readmission for a scheduled procedure; and (3) admissions for acute illness or for complications of care are never planned [6]. The hospital, which is part of a larger University Health System Consortium, defines a readmission as planned if the admission paperwork is processed more than 24 hours before the patient returns to the hospital [14]. The authors defined a "clinical" planned readmission as when the index discharge summary clearly indicated a planned future admission.

\section{Statistical Analysis}

Demographic information between readmitted and nonreadmitted patients was summarized with means (SD), median (interquartile range), and frequency (percentage), and statistical significance was assessed with t-tests, Wilcoxon rank-sum test, or chi-square tests when appropriate (Table 1). All eight subspecialty groups (foot/ankle, hand, joints, pediatrics, spine, sports, trauma, and tumor) were summarized between the two groups and it was found that the only statistical differences in 30-day readmission between groups were in the largest subspecialties, joints and trauma. The remaining subspecialties were consistent across groups and were therefore grouped together and called "other."
Associations with readmission were assessed with univariate and then multivariate logistic regression controlling for age, gender, and insurance type. Results from the logistic regression models were reported as odds ratio (OR) and $95 \%$ CI. Lastly, we constructed a best-fit model by using a forward stepwise selection process with all variables included in Table 1. A receiver operating characteristic (ROC) curve was constructed to assess the strength of association of the best-fit model.

The authors' determination of planned versus unplanned was compared with two other definitions that were recorded in the database. These three definitions (clinical, hospital, and CMS) were analyzed for agreement by using two kappa statistic methods, Light's and Fleiss', for multiple raters $[10,17]$.

\section{Results}

\section{Demographics}

During the 24-month time period, there were 4792 discharges. A 30-day readmission was identified in 202 (4\%; 95\% confidence interval $[\mathrm{CI}], 3.8-4.8)$ patients, whereas 4590 discharged patients were not readmitted (Table 1). In the patient cohort without readmission, the mean age was $54 \pm 20$ years. The trauma patients had a bimodal age distribution with a smaller peak in the third decade of life and a larger peak in the eighth decade. There were 2335 $(51 \%)$ men and $4191(94 \%)$ of the patients were white. More than half of the nonreadmitted patients $(60 \%)$ had private insurance. The joints subspecialty cared for 1885 $(41 \%)$ of the nonreadmitted patients. In the readmission cohort, the mean age was $56 \pm 20$ years. There were 105 (52\%) men and $185(92 \%)$ of the patients were white. Combining Medicare and Medicaid, 94 (47\%) had public insurance. The most common subspecialty was trauma, which treated 77 (39\%) patients. All of these demographics showed no association with 30-day readmission except for subspecialty.

\section{Differences in 30-day Readmission Rate by} Subspecialty

The overall 30-day readmission rate was $4 \%$ (95\% CI, 3.84.8). Joint arthroplasty and trauma services accounted for the majority of discharges with 1943 and 1207, respectively (Table 2). Spine also had a high number of discharges at 927, whereas foot and ankle had the fewest discharges at 35 . Hand and upper extremity had the highest 30-day readmission rate (5.4\% [four of 74]) but also had the second lowest number of discharges (Fig. 1). Tumor 
Table 1. Patient characteristic information and the relationship to 30-day readmission

\begin{tabular}{|c|c|c|c|}
\hline \multirow[t]{2}{*}{ Characteristic } & \multicolumn{2}{|c|}{ 30-day readmission status of 4792 patients } & \multirow[b]{2}{*}{$\mathrm{p}$ value } \\
\hline & Readmission $(\mathrm{n}=202)$ & No readmission $(n=4590)$ & \\
\hline Mean age (years) (mean \pm SD) & $56 \pm 20$ & $54 \pm 20$ & 0.146 \\
\hline \multicolumn{4}{|l|}{$\operatorname{Sex}$} \\
\hline Male & $105(52 \%)$ & $2335(51 \%)$ & \multirow[t]{2}{*}{0.813} \\
\hline Female & $97(48 \%)$ & $2255(49 \%)$ & \\
\hline \multicolumn{4}{|l|}{ Heritage } \\
\hline White & $185(92 \%)$ & $4191(94 \%)$ & \multirow[t]{7}{*}{0.175} \\
\hline Black & $5(2 \%)$ & $134(3 \%)$ & \\
\hline Native American & $2(1 \%)$ & $11(0.2 \%)$ & \\
\hline Asian & $2(1 \%)$ & $41(1 \%)$ & \\
\hline Hispanic & $5(3 \%)$ & $79(2 \%)$ & \\
\hline Multi & $1(0.5 \%)$ & $16(0.4 \%)$ & \\
\hline Pacific Islander & $1(0.5 \%)$ & $5(0.1 \%)$ & \\
\hline \multicolumn{4}{|l|}{ Insurance } \\
\hline Private & $108(54 \%)$ & $2730(60 \%)$ & \multirow[t]{3}{*}{0.138} \\
\hline Medicare & $82(41 \%)$ & $1552(34 \%)$ & \\
\hline Medicaid & $12(6 \%)$ & $308(7 \%)$ & \\
\hline Mean BMI $\left(\mathrm{kg} / \mathrm{m}^{2}\right)($ mean $\pm \mathrm{SD})$ & $30 \pm 8$ & $30 \pm 7$ & 0.122 \\
\hline \multicolumn{4}{|l|}{ ASA score } \\
\hline 1 & $20(11 \%)$ & $642(15 \%)$ & \multirow[t]{5}{*}{$<0.001^{*}$} \\
\hline 2 & $99(55 \%)$ & $2932(67 \%)$ & \\
\hline 3 & $52(29 \%)$ & $794(18 \%)$ & \\
\hline 4 & $7(4 \%)$ & $33(1 \%)$ & \\
\hline 5 & $1(1 \%)$ & $0(0 \%)$ & \\
\hline Median (interquartile range) length of stay (days) ${ }^{\dagger}$ & $3(3-6)$ & $3(2-4)$ & $<0.001^{*}$ \\
\hline \multicolumn{4}{|l|}{ Discharge disposition } \\
\hline Home & $76(28 \%)$ & $2175(47 \%)$ & \multirow[t]{4}{*}{$<0.001^{*}$} \\
\hline Home health & $49(25 \%)$ & $1119(24 \%)$ & \\
\hline SNF & $63(32 \%)$ & $1226(27 \%)$ & \\
\hline Other & $12(6 \%)$ & $70(2 \%)$ & \\
\hline \multicolumn{4}{|l|}{ Subspecialty } \\
\hline Joints & $56(28 \%)$ & $1885(41 \%)$ & \multirow[t]{3}{*}{$<0.001^{*}$} \\
\hline Trauma & $77(39 \%)$ & $1158(25 \%)$ & \\
\hline Other & $66(33 \%)$ & $1547(34 \%)$ & \\
\hline
\end{tabular}

* Statistically significant; ${ }^{\dagger}$ length of stay refers to index admission for surgery, not readmission. BMI = body mass index; ASA = American Society of Anesthesiologists; SNF = skilled nursing facility.

had the next highest readmission rate at $4.2 \%$ (six of 143). All other subspecialties had a 30-day readmission rate between 3-4\% except joints (2.8\% [55 of 1943]) and foot and ankle ( $0 \%$ [zero of 35]).

\section{Risk Factors}

Length of stay (OR, 1.10 per day [95\% CI, 1.07-1.14], p < 0.001), ASA score (OR, 1.89 per point [95\% CI, 1.442.46], $\mathrm{p}<0.001)$, discharge to "other" (OR, 4.91 [95\% CI, 2.43-9.21], $\mathrm{p}<0.001$ ), and care under trauma (OR, 2.55
[95\% CI, 1.77-3.68], p < 0.001) or "other" (OR, 1.65 [95\% CI, 1.13-2.40], $\mathrm{p}=0.009$ ) as compared with joint subspecialty were associated with increased risk of readmission after controlling for age, gender, and insurance type through multivariate analysis (Table 3). Initial univariate analysis showed that for each additional ASA level, a patient was 1.81 times more likely to be readmitted within 30 days ( $p<0.001)$, and this finding was also seen when controlling for age, gender, and insurance type in a multivariate analysis. Length of stay also showed an association with increased 30-day readmission. Each additional day in the hospital increased the likelihood of 
readmission by $10 \%$ (multivariate $\mathrm{p}$ value $<0.001$ ). Discharge to "other" compared with home demonstrated a univariate OR of $4.91(\mathrm{p}<0.001)$, and this was also seen in the multivariate analysis. Patients treated by a trauma subspecialist were 2.55 times more likely to be readmitted within 30 days as compared with joints $(\mathrm{p}<0.001)$ on multivariate analysis. Patients treated by any subspecialty other than joints or trauma had an increased chance of readmission (multivariate $\mathrm{OR}, 1.65 ; \mathrm{p}=0.009$ ).

The best-fit model found that ASA score, length of stay, discharge location, and subspecialty all contribute to the optimized model (Table 4), thus confirming the results of the multivariate analysis. The ROC curve demonstrates an area under the curve of 0.674 (Fig. 2).

Table 2. Unplanned readmission rate by subspecialty

\begin{tabular}{lcc}
\hline Subspecialty & Discharges & Unplanned readmissions \\
\hline Hand and upper extremity & 74 & $4(5.4 \%)$ \\
Tumor & 143 & $6(4.2 \%)$ \\
Trauma & 1207 & $46(3.8 \%)$ \\
Pediatrics & 308 & $11(3.6 \%)$ \\
Spine & 927 & $30(3.2 \%)$ \\
Sports & 155 & $5(3.2 \%)$ \\
Joints & 1943 & $55(2.8 \%)$ \\
Foot and ankle & 35 & $0(0 \%)$ \\
Total & 4792 & $157(3.4 \%)$ \\
\hline
\end{tabular}

\section{Readmission Causes}

Of the 202 readmitted patients, chart review identified 42 $(21 \%)$ as being clinically planned readmissions, $93(46 \%)$ as unplanned surgical readmissions, and $67(33 \%)$ as unplanned medical readmissions (Fig. 3).

Table 3. Multivariate logistic regression analysis of risk factors of 30-day readmission

\begin{tabular}{llr}
\hline Risk factor & \multicolumn{2}{l}{ Multivariate analysis* } \\
\cline { 2 - 3 } & OR (95\% CI) & p value \\
\hline ASA score & $1.89(1.44-2.46)$ & $<0.001^{*}$ \\
Length of stay & & $<0.001^{*}$ \\
Discharge location & $1.10(1.07-1.14)$ & \\
Home & Reference & 0.295 \\
Home health & $1.23(0.83-1.81)$ & 0.172 \\
SNF & $1.33(0.88-2.02)$ & $<0.001^{*}$ \\
Other & $4.91(2.43-9.21)$ & \\
Subspecialty & & $<0.001^{*}$ \\
Joints & Reference & $0.009^{*}$ \\
Trauma & $2.55(1.77-3.68)$ & \\
Other & $1.65(1.13-2.40)$ & \\
\hline
\end{tabular}

* Controlling for age, gender, and insurance type; ${ }^{\dagger}$ length of stay refers to index admission for surgery not the readmission "statistically significant; $\mathrm{OR}=$ odds ratio; $\mathrm{CI}=$ confidence interval; $\mathrm{ASA}=$ American Society of Anesthesiologists; SNF = skilled nursing facility.
Fig. 1 The unplanned 30-day readmission rate ranges from $0 \%$ to $5.4 \%$ when analyzed by subspecialty. UE = upper extremity.

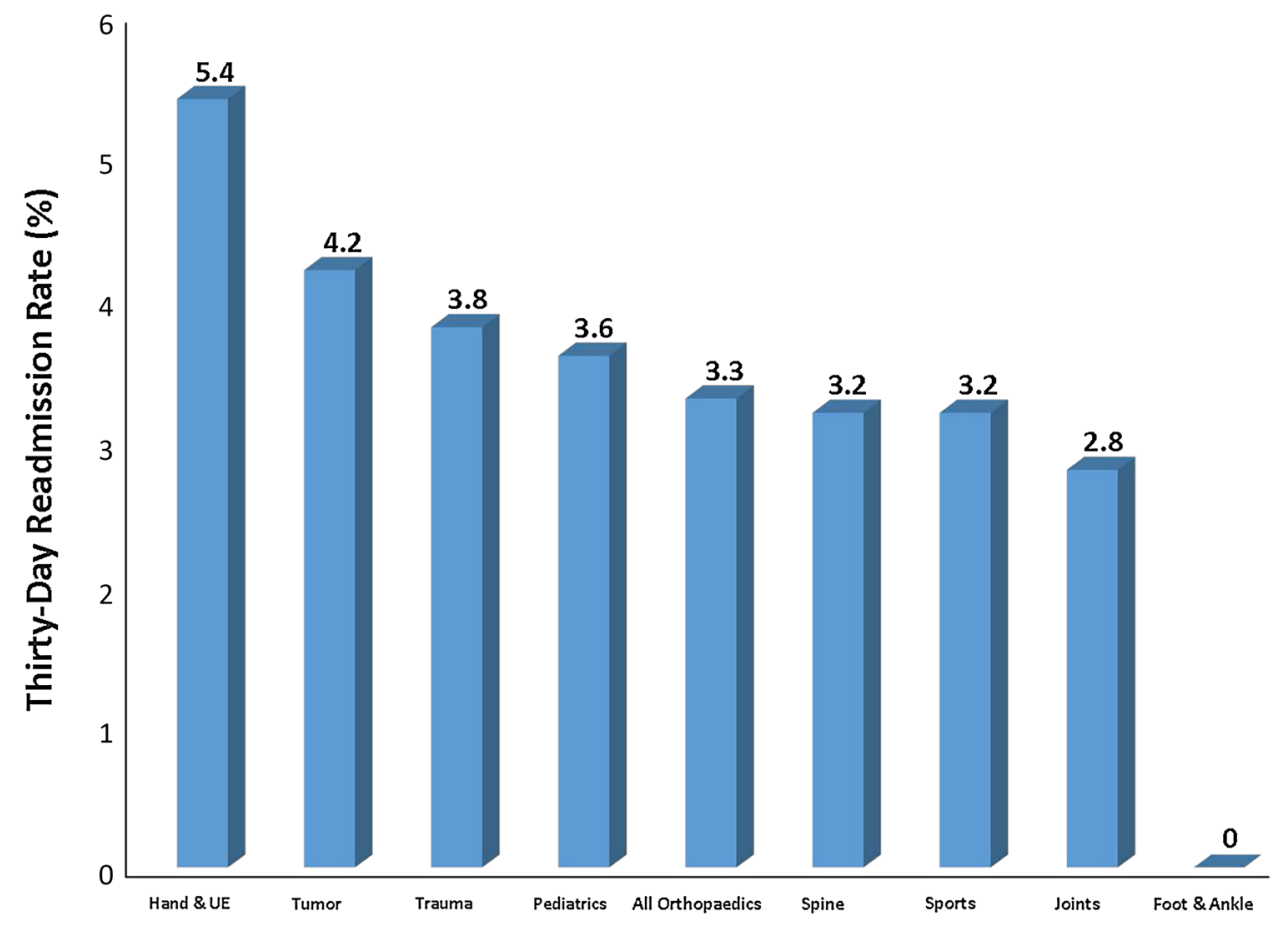


Table 4. Best-fit model of risk factors of 30-day readmission

\begin{tabular}{|c|c|c|}
\hline Risk factor & OR $(95 \% \mathrm{CI})$ & $\mathrm{p}$ value \\
\hline ASA score & $1.64(1.28-2.11)$ & $<0.001 *$ \\
\hline Length of stay & $1.07(1.03-1.11)$ & $<0.001 *$ \\
\hline \multicolumn{3}{|c|}{ Discharge location } \\
\hline Home & Reference & \\
\hline Home health & $1.69(1.06-2.66)$ & $0.025^{*}$ \\
\hline SNF & $1.30(0.84-2.01)$ & 0.232 \\
\hline Other & $2.54(1.11-5.31)$ & $0.019 *$ \\
\hline \multicolumn{3}{|l|}{ Subspecialty } \\
\hline Joints & Reference & \\
\hline Trauma & $2.25(1.46-3.47)$ & $<0.001 *$ \\
\hline Other & $1.69(1.08-2.64)$ & $0.021 *$ \\
\hline
\end{tabular}

* Statistically significant; $\mathrm{OR}=$ odds ratio; $\mathrm{CI}$ = confidence interval; ASA = American Society of Anesthesiologists; SNF = skilled nursing facility.

\section{Best Fit Model ROC Curve}

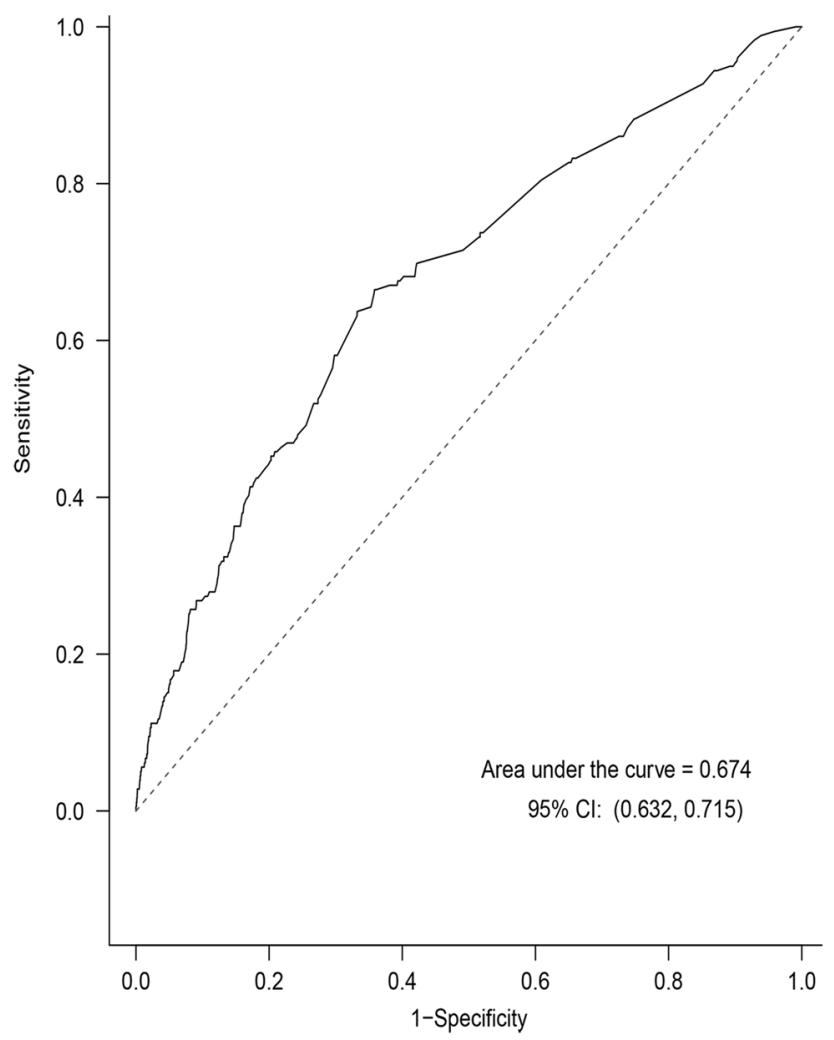

Fig. 2 The ROC curve represents the best-fit model based on ASA score, length of stay, discharge location, and subspecialty. The area under curve of 0.674 shows moderate association. LOS $=$ length of stay.

Combining both surgical and medical yielded 160 unplanned 30-day readmissions. This represented 3\% of the original 4792 orthopaedic discharges in the 24-month

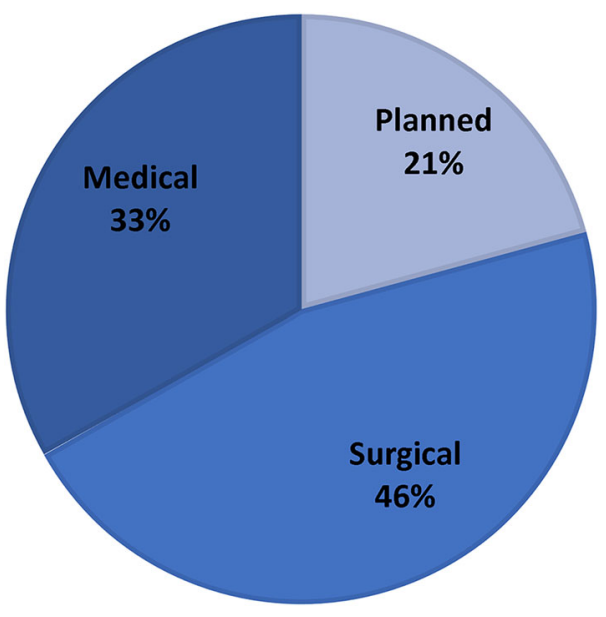

Fig. 3 Causes of 30-day readmission for 202 patients are medical, surgical, or planned.

enrollment period. Surgical causes accounted for 58\% (93 of 160) of unplanned readmissions, whereas medical causes accounted for the remaining 42\% (67 of 160). The 93 surgical readmissions were divided into three categories: surgical site, surgery, and medication/equipment (Table 5). The 67 medical readmissions were divided into nine body systems (Fig. 4). Most surgical causes relate to the surgical site (38\% [35 of 93]). Most medical causes were gastrointestinal (18\% [12 of 67]) and other (25\% [17 of 67]) (Table 6).

Agreement of Definitions of 'Planned' and 'Unplanned' Readmissions

Assessing all three definitions for planned and unplanned readmissions showed poor agreement (0.120) (Table 7). Clinical and CMS had the greatest two-variable agreement $($ kappa $=0.225$; Table 7), clinical to hospital had the next highest agreement at 0.163 , and hospital to CMS had the lowest agreement at 0.072 . Of the 202 readmissions, 42 (21\%) were planned by clinical definition, $61(30 \%)$ by hospital definition, and $12(6 \%)$ by CMS definition (Table 8).

\section{Discussion}

Decreased readmissions signifies better outcomes for patients. The 30-day readmission rate is thus an important quality metric that also carries financial implications as a result of its use in the CMS reimbursement program. First we set out to determine the readmission rate for each subspecialty to find out if any services may be unequally affected by the reimbursement program. We also sought 
Table 5. Surgical causes of unplanned readmission*

\begin{tabular}{llc}
\hline Category & Readmission cause & Frequency \\
\hline Surgical site & Surgical site Infection & $35(38 \%)$ \\
& Threatened wound & $11(12 \%)$ \\
& Hematoma & $8(9 \%)$ \\
& Dehiscence & $5(5 \%)$ \\
& Pin site infection & $2(2 \%)$ \\
& Seroma & $1(1 \%)$ \\
Total & $62(67 \%)$ \\
& Dural tear & $8(9 \%)$ \\
& Anemia & $3(3 \%)$ \\
& Dislocation & $3(3 \%)$ \\
& Revision & $3(3 \%)$ \\
& Dead space & $1(1 \%)$ \\
& Periprosthetic Fracture & $1(1 \%)$ \\
& Rescheduled & $1(1 \%)$ \\
& Total & $20(22 \%)$ \\
Medication/equipment & Pain & $4(4 \%)$ \\
& Allergic reaction & $3(3 \%)$ \\
& Drug problem & $3(3 \%)$ \\
& Brace problem & $1(1 \%)$ \\
& Total & $11(12 \%)$ \\
& & $93(100 \%)$ \\
& & 10
\end{tabular}

* If multiple causes of readmission, priority given to the principal admitting diagnosis on readmission.

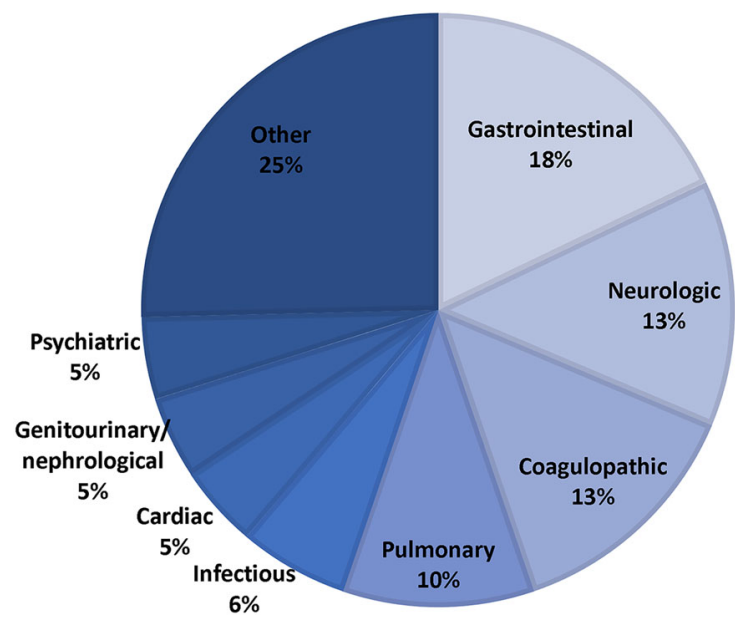

Fig. 4 Medical causes of unplanned readmission are divided into nine subcategories. $\mathrm{GU}=$ genitourinary; $\mathrm{GI}=$ gastrointestinal.

the risk factors and causes of readmission to add to the body of published data on this topic and identify aims for future studies on quality improvement. Additionally, the definitions of planned and unplanned readmission, to our knowledge, had not been assessed for agreement and warranted further investigation if they are to influence repayment. We found that subspecialties involved in more
Table 6. Medical causes of unplanned readmission*

\begin{tabular}{lc}
\hline Cause & Frequency \\
\hline Gastrointestinal & $12(18 \%)$ \\
Neurologic & $9(13 \%)$ \\
Coagulopathic & $9(13 \%)$ \\
Pulmonary & $7(10 \%)$ \\
Infectious & $4(6 \%)$ \\
Cardiac & $3(5 \%)$ \\
Genitourinary/nephrological & $3(5 \%)$ \\
Psychiatric & $3(5 \%)$ \\
Other & $17(25 \%)$ \\
\hline
\end{tabular}

* If multiple causes of readmission, priority given to the principal admitting diagnosis on readmission.

Table 7. Agreement analysis for three definitions of planned

\begin{tabular}{ll}
\hline Definitions & Kappa statistic \\
\hline Clinical-CMS & 0.225 \\
Clinical-hospital & 0.163 \\
Hospital-CMS & 0.072 \\
Fleiss-all 3 definitions & 0.120 \\
Light-all 3 definitions & 0.153 \\
\hline
\end{tabular}

CMS $=$ Centers for Medicare \& Medicaid Services.

Table 8. Planned 30-day readmissions by definition $(\mathrm{n}=202)$

\begin{tabular}{lll}
\hline Definition & Planned readmissions & Unplanned readmissions \\
\hline Clinical & $42(21 \%)$ & $160(79 \%)$ \\
Hospital & $61(30 \%)$ & $141(70 \%)$ \\
CMS & $12(6 \%)$ & $190(94 \%)$ \\
\hline
\end{tabular}

CMS $=$ Centers for Medicare \& Medicaid Services.

nonelective, emergent conditions (such as trauma, tumor, and spine) have higher readmission rates. The independent risk factors for readmission are length of stay and ASA score, whereas the most common cause of readmission is surgical site infection. Finally, the definition of unplanned readmission used by CMS for repayment does not agree with our clinical definition and therefore may be penalizing instances of safe and cost-effective care.

This study has several limitations. First, the hospital quality database does not track readmissions to outside hospitals. Therefore, we believe that our 30-day readmission rate is lower than the true rate. Furthermore, the database was unable to track patients with orthopaedic injuries who were admitted to the medicine or general surgery trauma service. These patients would be primed for readmission because they are often ones who are sent home in external fixators with planned readmission to orthopaedics. Grouping subspecialties based on the surgeon may 
have also introduced error if, for example, a joint surgeon completed a trauma surgery while on call. Third, although the records of the readmission cohort were explored for demographic and comorbidity data, we relied on coding to determine this information for the patients who did not have a 30-day readmission. The International Classification of Diseases, $9^{\text {th }}$ Revision coding has been shown to underreport chronic and comorbid conditions [11]; therefore, the data may not contain the true comorbidities of the control group. There is no gold standard definition of planned readmission, and although we feel our clinical definition improves on the CMS and hospital definitions, it may not represent the true rate. Finally, our study was conducted at an academic tertiary care center and the results may not be generalizable to a broader orthopaedic patient population.

We report an all-cause 30-day readmission rate of $4 \%$. When compared with joints, both the trauma and "other" subspecialties showed a correlation with increased rates of 30-day readmission. There could be many explanations for this finding. Arthroplasty patients' hospital stays typically are well planned, and followup is scheduled in advance of surgery. Close followup has been shown to reduce readmissions [4]. Also, joint patients are more likely to be medically optimized before surgery, limiting the number of patients with excessive or poorly compensated or controlled comorbidities. Other subspecialties perform more trauma-related and fewer elective surgeries, thus decreasing their ability to select against patients with an extensive medical history or preemptively schedule followup appointments. This finding is important because a hospital, under monetary reimbursement pressures by the CMS, may elect to deemphasize or eliminate those service lines that incur higher readmission rates. Access to care may be jeopardized and patient outcomes may be at risk if, for example, a trauma center is eliminated and patients must go further for their care. Our study suggests that the current repayment structure favors orthopaedic centers that do more elective procedures than trauma care.

Our study suggests that ASA score and length of stay are independent predictors of all-cause 30-day readmission. Length of stay has been well studied and shown to be a risk factor for readmission in the orthopaedic literature $[8,9$, $22,23,27]$. We believe this is because patients typically have longer admissions if they have had a more intense procedure or have other medical conditions requiring treatment, both of which could impart a greater risk of 30day readmission. We recommend that future studies examine the benefits of close monitoring and rigorous followup on the readmission rate of patients with extended lengths of stay. The correlation of ASA score to increased 30-day readmissions aligns with results reported in the orthopaedic spine literature $[3,16,23,25]$. We believe that an increased ASA score aligns with increased comorbidities, thus increasing the likelihood of 30-day readmission. Discharge to "other" was also found to be highly predictive of 30-day readmission. The category "other" included hospice, long-term acute care hospital, or jail. Patients being discharged to hospice or a long-term acute care hospital are in frail condition and are more likely to return to the hospital for conditions related to their surgery or other medical complaints. Although our study and the CMS repayment programs focus on short-term outcomes, future studies should examine these risk factors in regard to longterm outcomes because they may impact morbidity, mortality, and healthcare costs.

Our study finds that approximately $60 \%$ of unplanned 30-day readmissions are the result of surgical complications. This is low compared with other orthopaedic studies $[9,27]$. As we would expect with a low incidence of surgical readmission, our medical 30-day readmission rate of $42 \%$ is high when compared with other studies [18, 20, 23]. The most common cause of surgical readmission is surgical site infection. Our rate appears to be consistent with other studies reporting on a variety of orthopedic procedures $[9$, 28]. The threatened wound category represents $7 \%$ of the unplanned readmissions. These are cases in which the patient was readmitted for intravenous antibiotics; however, wound cultures were negative and the Centers for Disease Control and Prevention definition of surgical site infection was not met. Theoretically, these patients could be treated in an outpatient setting and may not require admission. Alternatively, treatment could occur in an emergency department 23-hour observation unit. Eliminating readmissions for threatened wounds would decrease unplanned readmissions by $9 \%$ or absolute $0.3 \%$. Therefore, we envision the development of protocols to manage these threatened wound patients in the clinic or primary care setting as an avenue of readmission reduction. The causes of medical readmission seem diffuse. The most common causes are unrelated trauma, pulmonary embolism, and gastrointestinal bleed. From these data, we are unable to single out a specific cause of readmission that could be targeted by a quality improvement program. Rather, we suggest that more generalizable methods be studied to comprehensively lower the readmissions as a result of other medical causes. One example of this would be followup telephone calls after discharge, which have been validated as a means of reducing readmission in medicine patients but not yet in orthopaedic patients [13].

The definitions of a planned readmission as articulated by CMS, the hospital, and the providers showed poor agreement with one another in this study. This represents a serious problem because providers are incentivized to provide care that may not be best for the patient. If, for example, a patient is sent home on conservative 
management but the disease progresses and ultimately requires surgery in the next 30 days, CMS penalizes the readmission as "unplanned." Under this system, surgeons may be dissuaded from trialing conservative management and thus recommend surgery more often. This is not a safe practice. The shortcomings of using coding to determine quality metrics have been reported previously $[1,14,19$, 24]. We found that the CMS definition of planned, which is currently used for withholding reimbursements from hospitals, labeled 30 readmissions that our authors felt were safe, best-practice management (as defined by the clinical definition) as unplanned readmissions. The CMS withheld payments from 2213 hospitals in fiscal year 2013, totaling USD 280 million in readmission penalties [7]. The findings of this study suggest that hospitals are being penalized for planned readmissions, which represent responsible, costeffective care.

Our study demonstrates the differences in readmission rate by subspecialty within orthopaedics. This suggests that nonelective services such as trauma and tumor bolster the 30-day readmission rate and are therefore the largest contributor to repayment penalties. This is an important finding because minimizing these services as a result of financial incentives could greatly limit access to care for patients who need it most. The results of our study also identify potential avenues of readmission reduction. We would like to develop a consensus on the treatment of patients with threatened wounds. Minimizing readmissions for surgical site complications could greatly reduce unplanned readmissions. We have also implemented a telephone followup program for all patients discharged to a SNF. We feel this intervention may reduce 30-day readmissions in that population, but future studies will need to evaluate this in both the SNF and home discharge populations. Finally, we believe that our study, and others like it, have adequately demonstrated the pitfalls of using coding to determine quality metrics. From a hospital standpoint, we propose that an option be added to charting so that a physician can clearly identify when a patient will be returning for a planned readmission. From a CMS standpoint, we propose that they broaden their definition of planned to include patients who are returning for staged procedures. With these adjustments, there could be both a decrease in 30-day readmissions as well as a decrease in data coding discrepancies.

\section{References}

1. Amin BY, Tu TH, Schairer WW, Na L, Takemoto S, Berven S, Deviren V, Ames C, Chou D, Mummaneni PV. Pitfalls of calculating hospital readmission rates based on nonvalidated administrative data sets. J Neurosurg Spine. 2013;18:134-138.
2. Barnett ML, Hsu J, McWilliams J. Patient characteristics and differences in hospital readmission rates. JAMA Intern Med. 14 Sep 2015 [Epub ahead of print].

3. Basques BA, Varthi AG, Golinvaux NS, Bohl DD, Grauer JN. Patient characteristics associated with increased postoperative length of stay and readmission after elective laminectomy for lumbar spinal stenosis. Spine (Phila Pa 1976). 2014;39:833-840.

4. Brooke BS, Stone DH, Cronenwett JL, Nolan B, DeMartino RR, MacKenzie TA, Goodman DC, Goodney PP. Early primary care provider follow-up and readmission after high-risk surgery. JAMA Surg. 2014;149:821-828.

5. Centers for Medicare \& Medicaid Services. Comprehensive Care for Joint Replacement. September 2015. Available at: https://www. cms.gov/Newsroom/MediaReleaseDatabase/Fact-sheets/2015Fact-sheets-items/2015-07-09.html. Accessed September 20, 2015.

6. Centers for Medicare \& Medicaid Services. Planned readmission algorithm-version 2.1. March 2013. July 20, 2014. Available at: http://www.cms.gov/Medicare/Quality-Initiatives-Patient-AssessmentInstruments/HospitalQualityInits/Measure-Methodology.html. Accessed June 12, 2015.

7. Centers for Medicare \& Medicaid Services. Readmission Reduction Program. April 30, 2014. Available at: https://www.cms.gov/ medicare/medicare-fee-for-service-payment/acuteinpatientpps/ readmissions-reduction-program.html. Accessed June 29, 2014.

8. Clement RC, Derman PB, Graham DS, Speck RM, Flynn DN, Levin LS, Fleisher LA. Risk factors, causes, and the economic implications of unplanned readmissions following total hip arthroplasty. J Arthroplasty. 2013;28(Suppl):7-10.

9. Dailey EA, Cizik A, Kasten J, Chapman JR, Lee MJ. Risk factors for readmission of orthopaedic surgical patients. J Bone Joint Surg Am. 2013;95:1012-1019.

10. Fleiss JL. Measuring nominal scale agreement among many raters. Psychol Bull. 1971;76:378-382.

11. Garvin JH, Redd A, Bolton D, Graham P, Roche D, Groeneveld P, Leecaster M, Shen S, Weiner MG. Exploration of ICD-9-CM coding of chronic disease within the Elixhauser Comorbidity Measure in patients with chronic heart failure. Perspect Health Inf Manag. 2013;10:1b.

12. Gu Q, Koenig L, Faerberg J, Steinberg CR, Vaz C, Wheatley MP. The Medicare Hospital Readmissions Reduction Program: potential unintended consequences for hospitals serving vulnerable populations. Health Serv Res. 2014;49:818-837.

13. Harrison JD, Auerbach AD, Quinn K, Hynoch E, Mourad M. Assessing the impact of nurse post-discharge telephone calls on 30-day hospital readmission rates. J Gen Intern Med. 2014;29:1519-1525.

14. Hechenbleikner EM, Makary MA, Samarov DV, Bennett JL, Gearhart SL, Efron JE, Wick EC. Hospital readmission by method of data collection. J Am Coll Surg. 2013;216:1150-1158.

15. Jencks SF, Williams MV, Coleman EA. Rehospitalizations among patients in the Medicare fee-for-service program. $N$ Engl $J$ Med. 2009;360:1418-1428.

16. Kim BD, Smith TR, Lim S, Cybulski GR, Kim JY. Predictors of unplanned readmission in patients undergoing lumbar decompression: multi-institutional analysis of 7016 patients. $J$ Neurosurg Spine. 2014;20:606-616.

17. Landis JR, Koch GG. The measurement of observer agreement for categorical data. Biometrics. 1977;33:159-174.

18. Lovecchio F, Hsu WK, Smith TR, Cybulski G, Kim B, Kim JY. Predictors of thirty-day readmission after anterior cervical fusion. Spine (Phila Pa 1976). 2014;39:127-133.

19. McCormack R, Michels R, Ramos N, Hutzler L, Slover JD, Bosco JA. Thirty-day readmission rates as a measure of quality: causes of readmission after orthopedic surgeries and accuracy of administrative data. J Healthc Manag. 2013;58:64-76; discussion 76-77. 
20. McCormack RA, Hunter T, Ramos N, Michels R, Hutzler L, Bosco JA. An analysis of causes of readmission after spine surgery. Spine (Phila Pa 1976). 2012;37:1260-1266.

21. Medicare Payment Advisory Commission (US). Report to the Congress: reforming the delivery system. Medicare Payment Advisory Commission, June 2008. Available at: http://www.medpac.gov/documents/ reports/Jun08_EntireReport.pdf. Accessed March 2, 2014.

22. Mesko NW, Bachmann KR, Kovacevic D, LoGrasso ME, O'Rourke C, Froimson MI. Thirty-day readmission following total hip and knee arthroplasty-a preliminary single institution predictive model. J Arthroplasty. 2014;29:1532-1538.

23. Pugely AJ, Martin CT, Gao Y, Mendoza-Lattes S. Causes and risk factors for 30-day unplanned readmissions after lumbar spine surgery. Spine (Phila Pa 1976). 2014;39:761-768.

24. Sacks GD, Dawes AJ, Russell MM, Lin AY, Maggard-Gibbons M, Winograd D, Chung HR, Tomlinson J, Tillou A, Shew SB, Hiyama DT, Cryer HG, Brunicardi FC, Hiatt JR, Ko C. Evaluation of hospital readmissions in surgical patients: do administrative data tell the real story? JAMA Surg. 2014;149:759-764.

25. Schaeffer JF, Scott DJ, Godin JA, Attarian DE, Wellman SS, Mather RC $3^{\text {rd }}$. The association of ASA class on total knee and total hip arthroplasty readmission rates in an academic hospital. $J$ Arthroplasty. 2015;30:723-727.

26. Schairer WW, Carrer A, Deviren V, Hu SS, Takemoto S, Mummaneni P, Chou D, Ames C, Burch S, Tay B, Sawyer A, Berven SH. Hospital readmission after spine fusion for adult spinal deformity. Spine (Phila Pa 1976). 2013;38:1681-1689.

27. Schairer WW, Sing DC, Vail TP, Bozic KJ. Causes and frequency of unplanned hospital readmission after total hip arthroplasty. Clin Orthop Relat Res. 2014;472:464-470.

28. Zmistowski B, Restrepo C, Hess J, Adibi D, Cangoz S, Parvizi J. Unplanned readmission after total joint arthroplasty: rates, reasons, and risk factors. J Bone Joint Surg Am. 2013;95:18691876. 